\title{
Ice-gas interactions during planet formation
}

\author{
Karin I. Öberg \\ Harvard-Smithsonian Center for Astrophysics \\ 60 Garden St, Cambridge, MA 02138 \\ email: koberg@cfa.harvard.edu
}

\begin{abstract}
Planets form in disks around young stars. In these disks, condensation fronts or snowlines of water, $\mathrm{CO}_{2}, \mathrm{CO}$ and other abundant molecules regulate the outcome of planet formation. Snowline locations determine how the elemental and molecular compositions of the gaseous and solid building blocks of planets evolve with distance from the central star. Snowlines may also locally increase the planet formation efficiency. Observations of snowlines have only become possible in the past couple of years. This proceeding reviews these observations as well as the theory on the physical and chemical processes in disks that affect snowline locations.
\end{abstract}

Keywords. astrochemistry, astrobiology, molecular processes

\section{Introduction}

Planets form in disks around young stars. These disks are composed of dust and gas, where both the dust and gas composition varies with disk radius and height. These variations come in two flavors. First, a combination of molecules inherited from the protostellar stage and ongoing chemistry in disks determines the composition of volatiles in different disk regions and at different times. Second, a balance between freeze-out and sublimation, where the sublimation rate is set by a combination of volatile binding energies on (icy) dust grains, the thermal structure and the flux of high-energy radiation, determines how specific volatiles are divided between gas and dust/ice phases (Öberg et al. 2011b). This balance is generally achieved at short time scales compared to chemical and dynamical timescales, but exceptions exist as discussed in $\S 3$.

In disks the main reservoirs of volatiles after hydrogen are expected to be water ice, $\mathrm{CO}_{2}$ and $\mathrm{CO}$ ice and gas. Apart from gas-phase $\mathrm{CO}$, the most abundant volatiles are effectively hidden from view. Ices are generally not possible to observe in disks, and $\mathrm{CO}_{2}$ has no strong rotational transitions because of its lack of a permanent dipole moment. The volatile composition in the preceding protostellar stage is better understood because of surveys of ice absorption spectra toward low and high-mass protostars where the protostar is used as the background IR source toward which ice absorption is measured. Based on such surveys, it is well established that water is the most important ice constituent, followed by $\mathrm{CO}$ and $\mathrm{CO}_{2}$ and trace amounts of $\mathrm{CH}_{3} \mathrm{OH}, \mathrm{CH}_{4}$ and $\mathrm{NH}_{3}$ (Gibb et al. 2004, Öberg et al. 2011a, Boogert et al. 2015). $\mathrm{N}_{2}$ is probably also an important ice constituent in the coldest lines of sight, but cannot be observed. Figure 1 illustrates the median ice composition as well as the range of compositions observed in different lines of sight. It is clear that for a disk that does not experience a major chemical evolution, $\mathrm{H}_{2} \mathrm{O}, \mathrm{CO}_{2}$ and $\mathrm{CO}$ will be the most important snowlines for carbon and oxygen containing species.

This proceeding summarizes recent successful attempts at observing snowlines of $\mathrm{H}_{2} \mathrm{O}$ and CO in protoplanetary disks using ALMA and infrared observations ( $(2)$. $\S 3$ reviews and discusses the processes that regulate snowline locations in disks. 


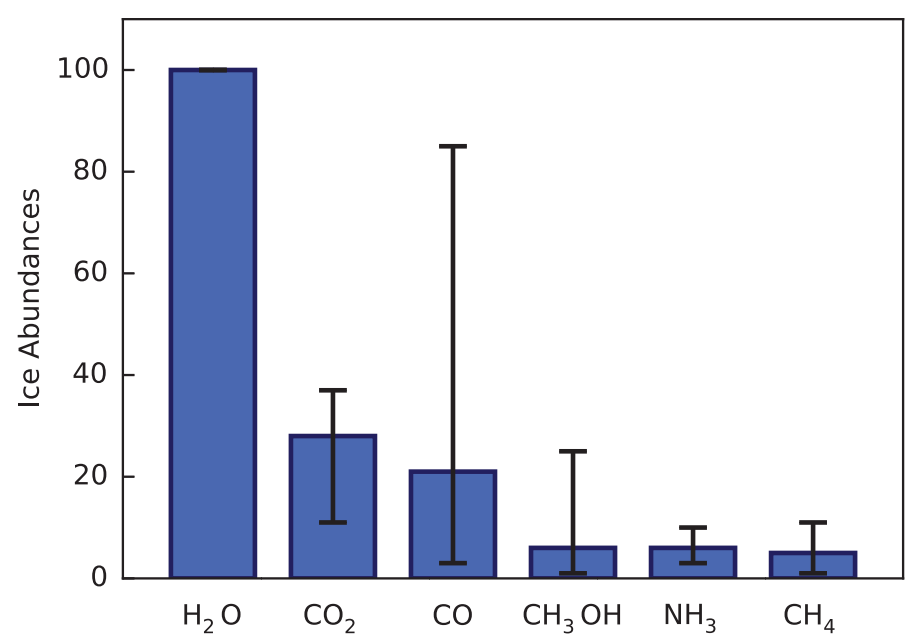

Figure 1. The median ice composition with respect to water toward low-mass protostars (blue bars). The black 'error bars' represent the minimum and maximum abundances observed with respect to water ice in low-mass protostellar lines of sight. Values are taken from Boogert et al. (2015).

\section{Observations of snowlines in disks}

Three disk snowlines have been observed during the past few years, the water snowline in the disk around TW Hya (Zhang et al. 2013), and the CO snowlines in the disks around TW Hya (Qi et al. 2013) and HD 163296 (Qi et al. 2011, Mathews et al. 2013, Qi et al. 2015). The water snowline toward TW Hya was extracted from infrared and far-infrared observations of water vapor emission lines. Based on detailed modeling of the lines, Zhang et al. (2013) found that water vapor in the disk is present in a narrow ring with the inner rim close to the $4 \mathrm{AU}$ dust hole in the disk. The outer 4.2 AU rim was interpreted as the water snowline.

The CO snowline has been resolved using millimeter interferometry, by direct CO observations and through imaging of other trace species whose chemistry is closely linked to $\mathrm{CO}$ freeze-out. Early attempts to use ${ }^{13} \mathrm{CO}$ observations to constrain the CO snowline location seems to have overestimated the CO snowline radius by 50-60 AU, highlighting the potential traps of this method (Qi et al. 2011). To avoid such traps, Qi et al. (2013) used $\mathrm{N}_{2} \mathrm{H}^{+}$to determine the $\mathrm{CO}$ snowline location in the TW Hya disk. $\mathrm{N}_{2} \mathrm{H}^{+}$ is expected to be anti-correlated with $\mathrm{CO}$ gas because $\mathrm{CO}$ gas inhibits formation and speeds up destruction of $\mathrm{N}_{2} \mathrm{H}^{+}$. The inner rim of $\mathrm{N}_{2} \mathrm{H}^{+}$emission should therefore mark the $\mathrm{CO}$ midplane snowline (Fig. 2). Another potential tracer of the $\mathrm{CO}$ snowline, $\mathrm{DCO}^{+}$ (Mathews et al. 2013) has since been found to have a more complicated relationship with $\mathrm{CO}$ freeze-out. This conclusion is based on comparison between $\mathrm{DCO}^{+}, \mathrm{N}_{2} \mathrm{H}^{+}$and $\mathrm{C}^{18} \mathrm{O}$ emission in the HD 163296 disk (Qi et al. 2015) as well as the presence of a pair of concentric $\mathrm{DCO}^{+}$rings in the disk of IM Lup (Öberg et al. 2015).

Based on the $\mathrm{N}_{2} \mathrm{H}^{+}$observations toward TW Hya and HD 163296, the CO snowline does not appear to occur at the same disk temperature in the two disks. Toward TW Hya, the derived CO snowline location corresponds to a disk midplane temperature of $17 \mathrm{~K}$, while in HD 163296 the CO snowline location corresponds to $25 \mathrm{~K}$ (Qi et al. 2013, Qi et al. 2015). These observations imply that there is not a single temperature that defines the $\mathrm{CO}$ snowline location in all disks, which should be the case if disk dynamics 


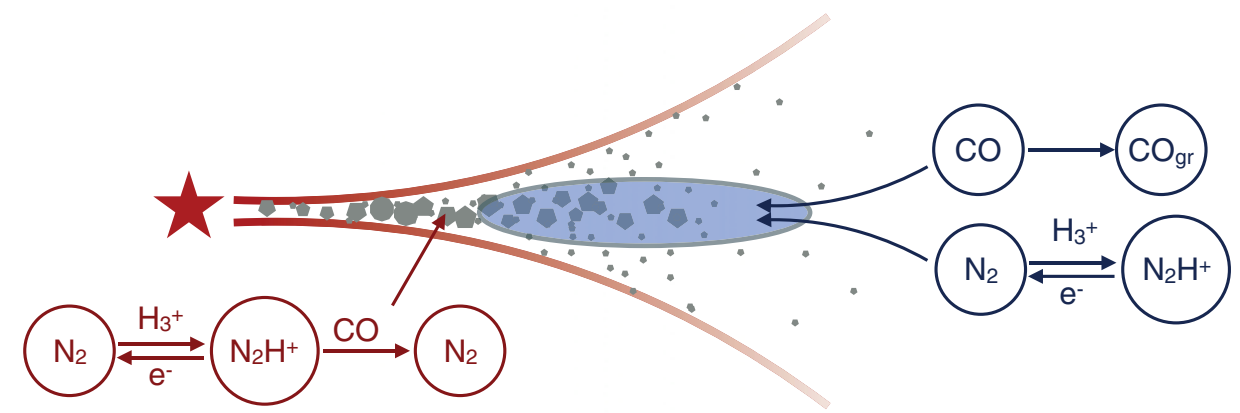

Figure 2. Abundant $\mathrm{N}_{2} \mathrm{H}^{+}$is only expected in disk regions where $\mathrm{CO}$ is depleted due to $\mathrm{CO}$ freeze-out, i.e. in the disk midplane exterior to the $\mathrm{CO}$ snowline (blue region). Interior to the $\mathrm{CO}$ snowline $\mathrm{N}_{2} \mathrm{H}^{+}$formation is inhibited by $\mathrm{CO}$ reactions with $\mathrm{H}_{3}^{+}$while $\mathrm{N}_{2} \mathrm{H}^{+}$is rapidly destroyed by $\mathrm{CO}$ reactions with $\mathrm{N}_{2} \mathrm{H}^{+}$. In the absence of $\mathrm{CO}, \mathrm{N}_{2} \mathrm{H}^{+}$is mainly destroyed by much slower electron recombination reactions.

is always slow compared to sublimation and if the ice structure and therefore ice binding energy is the same in all disks.

\section{Theory of snowline locations}

Figure 3 shows the $\mathrm{C} / \mathrm{O}$ ratio in gas and dust across a disk due to water, $\mathrm{CO}_{2}$ and CO snowlines, assuming a typical disk density and temperature profile, a static disk, a lack of chemical evolution, and standard assumptions on the binding energies of these three molecules (Öberg et al. 2011b). Disks are dynamic systems however, and they do chemically evolve and may present different volatile binding energies dependent on the local ice environment. Major snowlines may therefore occur at different disk midplane temperatures in different disks.

Fundamentally a snowline location is set by the balance of desorption (sublimation) and adsorption of a volatile. In a static disk, the temperature at which the adsorption overtakes desorption can be calculated if the density and binding energy are known. The dependence on density is weak (logarithmic), however (Hollenbach et al. 2009) and will at most change the snowline temperature by a few degrees for 'reasonable' disk midplane density profiles. The dependence on binding energy is stronger (linear), and the CO binding energy is known to depend sensitively on the local ice environment. In particular $\mathrm{CO}$ interactions with porous water ice can be almost twice as strong as the interaction between $\mathrm{CO}$ molecules in a pure $\mathrm{CO}$ ice. If $\mathrm{CO}$ is sufficiently abundant to form a pure $\mathrm{CO}$ ice on grains, the $\mathrm{CO}$ snowline should thus occur far out in the disk at locations with dust temperatures of $20 \mathrm{~K}$ or less (cf. TW Hya). In disks where CO is mixed with water ice, CO will, by contrast, begin to freeze out at $25-30 \mathrm{~K}$.

Ices can also desorb non-thermally. UV ice photodesorption is efficient (Fayolle et al. 2011). The presence of strong UV fields may push CO snowlines outward, corresponding to a lower freeze-out temperature than would be expected from a balance between adsorption and thermal desorption alone (Fig. 3). Finally, there are several dynamical processes that can affect snowline locations. Accretion flows and drift of grains can move snowlines inward by $\sim 50 \%$ compared to the expected locations in static disks (Piso et al. ApJ subm.), if grains have grown to $\mathrm{cm}$ size or larger. For these larger grains drift timescale are shorter than sublimation time scales. Considering the number of processes that can affect snowline locations, it is perhaps not strange that $\mathrm{CO}$ snowlines have been detected 

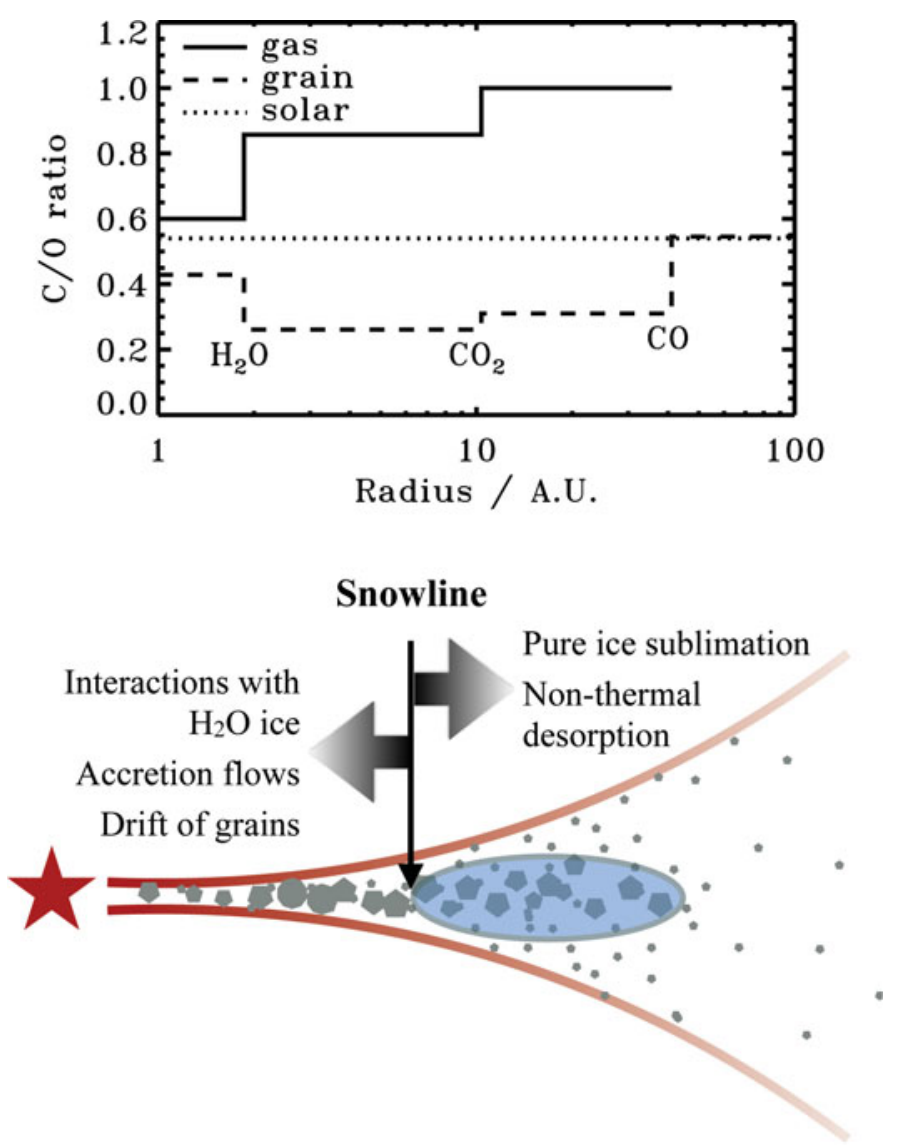

Figure 3. Top: The $\mathrm{C} / \mathrm{O}$ gas and dust ratio in a disk assuming a static disk and standard prescriptions for the balance between desorption and adsorption for the most abundant $\mathrm{C}$ and $\mathrm{O}$ volatiles. Bottom: The qualitative effects of some disk processes on snowline locations in disks.

at locations that correspond to different $\mathrm{CO}$ freeze-out temperatures. With more data it should be possible to tell whether chemical and dynamical effects regulate $(\mathrm{CO})$ snowline locations and thus how snowlines evolve during planet formation.

\section{References}

Collings, M. P., Dever, J. W., \& Fraser, H. J. 2003, ApJ, 583, 1058

Hollenbach, D. J., Kaufman, M. J., Bergin, E. A., \& Melnick, G. J. 2009, ApJ, 690, 1497

Mathews, G. S, Klaassen, P. D, Juhasz, A, et al. 2013, A\&A, 557, 132

Öberg K. I., Boogert A. C. A., Pontoppidan K. M. et al. 2011 ApJ, 740, 109

Öberg, K. I., Murray-Clay, R., Bergin, E. A. 2011 ApJL, 743, L16

Öberg, K. I., Furuya, K. and Loomis, R. 2015 ApJ, 810, 112

Qi, C., Öberg, K. I., Wilner, D. J. et al. 2013, Science, 341, 630

Qi, C., Öberg, K. I., Andrews, S. M. et al. 2015, eprint arXiv, 1510.00968

Zhang, K., Pontoppidan, K. M., Salyk, C., \& Blake, G. A. 2013, ApJ, 766, 82

Fayolle, E. C., Bertin, M., \& Romanzin, C. 2011, ApJL, 739, L36 